BMJ Open

Diabetes

Research

\& Care

\title{
Further improvement in glycemic control after switching from exenatide two times per day to exenatide once- weekly autoinjected suspension in patients with type 2 diabetes: 52-week results from the DURATION- NEO-1 study
}

\author{
Carol H Wysham (D) , ${ }^{1}$ Julio Rosenstock, ${ }^{2}$ Marion L Vetter, ${ }^{3}$ Hui Wang, ${ }^{4}$ \\ Elise Hardy, ${ }^{5}$ Nayyar lqbal ${ }^{6}$
}

To cite: Wysham $\mathrm{CH}$, Rosenstock J, Vetter ML, et al. Further improvement in glycemic control after switching from exenatide two times per day to exenatide once-weekly autoinjected suspension in patients with type 2 diabetes: 52 -week results from the DURATION-NEO-1 study. BMJ Open Diab Res Care 2020;8:e000773. doi:10.1136/ bmjdrc-2019-000773

\section{- Additional material is published online only. To view, please visit the journal online (http://dx.doi.org/10.1136/ bmjdrc-2019-000773).}

The 52-week results of this study have not been presented previously.

Received 22 July 2019 Revised 27 May 2020 Accepted 22 June 2020

Check for updates

C Author(s) (or their employer(s)) 2020. Re-use permitted under CC BY-NC. No commercial re-use. See rights and permissions. Published by BMJ.

For numbered affiliations see end of article.

Correspondence to Dr Carol H Wysham; cwysham@multicare.org

\section{ABSTRACT}

Introduction Investigate the effects of switching from two times per day exenatide to once-weekly exenatide administered by autoinjector (exenatide once-weekly suspension by autoinjector (QWS-Al)) or treatment with exenatide QWS-Al for 1 year.

Research design and methods In this phase III open-label study, adults with type 2 diabetes were randomized to receive exenatide QWS-Al ( $2 \mathrm{mg}$ ) or exenatide two times per day (5 mcg for 4 weeks, followed by $10 \mathrm{mcg}$ ) for 28 weeks. During a subsequent non-randomized 24-week extension, patients who received exenatide two times per day were switched to exenatide QWS-Al and those randomized to exenatide QWS-Al continued this treatment. Efficacy measures included changes from baseline in glycated hemoglobin (A1C), fasting plasma glucose (FPG), and body weight.

Results In total, 315 patients (mean baseline A1C of $8.5 \%$ ) completed the initial 28 weeks of randomized treatment with exenatide QWS-Al $(n=197)$ or exenatide two times per day $(n=118)$ and were included in the 24-week extension (mean A1C of $7.0 \%$ and $7.3 \%$, respectively, at week 28 ). From weeks 28-52, patients who switched from exenatide two times per day to exenatide QWS-Al had additional A1C reductions of approximately $0.5 \%$ (mean $\mathrm{A} 1 \mathrm{C}$ change from baseline of $-1.4 \%$ at week 52 ) and further reductions from baseline in FPG. Patients who continued exenatide QWSAl treatment for 52 weeks showed clinically relevant A1C reductions (mean $\mathrm{A} 1 \mathrm{C}$ change from baseline of $-1.3 \%$ at week 52). Body-weight reductions achieved through week 28 were sustained at week 52 in both groups. There were no unexpected safety concerns or changes in the safety profile among patients who switched from exenatide two times per day to exenatide QWS-Al or those who continued exenatide QWS-Al treatment for 52 weeks.

Conclusions Switching from exenatide two times per day to exenatide QWS-Al resulted in further $\mathrm{A} 1 \mathrm{C}$ reductions and maintenance of earlier decreases in body weight, while continued therapy with exenatide QWS-Al for 52 weeks

\section{Significance of this study}

What is already known about this subject?

- Switching from exenatide two times per day to an aqueous formulation of exenatide once weekly (QW) at 30 weeks in the DURATION-1 study resulted in further decreases in glycated hemoglobin (A1C) and sustained reductions in body weight over 52 weeks without additional safety concerns, while patients continuing on exenatide QW over 52 weeks maintained a stable $\mathrm{A} 1 \mathrm{C}$.

- A new non-aqueous, medium-chain triglyceridebased formulation of exenatide $Q W$, exenatide once-weekly suspension by autoinjector (QWS-Al), is more easily administered and more convenient to use than the aqueous formulation, but it is not known whether it will provide similar sustained results at 52 weeks.

What are the new findings?

- Switching from exenatide two times per day to exenatide QWS-Al resulted in further decreases in $\mathrm{A} 1 \mathrm{C}$ and maintenance of earlier reductions in body weight, while continuing with exenatide QWS-Al for 52 weeks maintained reductions in $\mathrm{A} 1 \mathrm{C}$ and body weight without new safety or tolerability concerns.

- These results confirm earlier findings that exenatide QWS-Al is more effective in lowering A1C than exenatide two times per day and demonstrate that patients treated with exenatide QWS-Al achieve clinically relevant $\mathrm{A} 1 \mathrm{C}$ reductions through 52 weeks of treatment.

maintained $\mathrm{A} 1 \mathrm{C}$ and body-weight reductions, without additional safety or tolerability concerns. Trial registration number NCT01652716. 


\section{Significance of this study}

How might these results change the focus of research or clinical practice?

- These results assist physicians and patients in understanding the potential outcomes of switching from the immediate-release formulation of exenatide (exenatide two times per day) to the more easily administered suspension formulation for autoinjection (exenatide QWS-Al) and also inform them of the potential benefits of continuing on exenatide QWS-Al for up to 52 weeks.

\section{INTRODUCTION}

Adherence (ie, taking medication as prescribed) to glucose-lowering therapy is often suboptimal among patients with type 2 diabetes, with evidence suggesting that at least $50 \%$ of patients do not conform to the recommended timing, dosage, and frequency of medication, particularly with respect to injectable therapies. ${ }^{1}$ In clinical practice, treatment persistence (ie, maintenance of therapy) with glucagon-like peptide-1 receptor agonists (GLP-1RAs) is suboptimal, with higher rates of discontinuation among therapies that require reconstitution before administration or more frequent administration; furthermore, patients may prefer injectable treatments that do not require handling of needles. ${ }^{2}$ Non-adherence and nonpersistence with glucose-lowering therapy are associated with worse clinical outcomes, including poorer long-term glycemic control, more diabetes-associated complications, greater healthcare resource use, and higher costs. ${ }^{1-5}$ The availability of efficacious treatments that are simple to administer is likely to increase treatment adherence and persistence. While patients with poor adherence may switch medications to find a more sustainable regimen, patients with good adherence and response to their current therapy may also consider switching to medications that are more convenient and easier to administer, particularly closely related formulations.

Exenatide is a first-in-class member of the GLP-1RA class of glucose-lowering therapies. It was first available in a two-times-per-day formulation, followed by a once-weekly (QW) aqueous formulation. The aqueous formulation of exenatide QW contains exenatide molecules packaged into biodegradable poly-(D,L-lactide-co-glycolide) microspheres and is reconstituted in an aqueous diluent before administration. ${ }^{6}$ Subsequently, a new QW formulation has been developed, in which exenatide is encapsulated in biodegradable microspheres containing exenatide $2 \mathrm{mg}$ suspended in medium-chain triglycerides (Miglyol 812) and administered by an autoinjector with a hidden needle (exenatide once-weekly suspension by autoinjector (QWS-AI)). Because the autoinjector does not require reconstitution or dose selection and requires fewer steps to administer than either the exenatide QW single-dose tray or dual-chamber pen, exenatide QWS-AI is more convenient and easier to use than the aqueous exenatide QW product. $^{7}$
The phase III, randomized, controlled, open-label DURATION-NEO-1 study compared the efficacy, safety, and tolerability of exenatide QWS-AI with exenatide two times per day. Results from the initial 28-week controlled treatment period showed that, compared with exenatide two times per day, exenatide QWS-AI produced a greater reduction in glycated hemoglobin (A1C) and comparable weight loss, and had improved gastrointestinal tolerability. ${ }^{8}$ We report the results of a 24-week extension phase of the DURATION-NEO-1 study, which studied the effects of switching to exenatide QWS-AI from exenatide two times per day and of continuing exenatide QWS-AI for 52 weeks.

\section{RESEARCH DESIGN AND METHODS}

Full details of the DURATION-NEO-1 study design have been previously published. ${ }^{8}$ Briefly, DURATION-NEO-1 enrolled adults ( $\geq 18$ years) with type 2 diabetes who were treated with diet and exercise alone or with a stable regimen of metformin, sulfonylurea, pioglitazone, or any combination of two of these agents, and had an A1C of $7.1 \%$ to $\leq 11.0 \%$. Patients were randomized in a $3: 2$ ratio to receive 28 weeks of treatment with either exenatide QWS-AI ( $2 \mathrm{mg})$ or exenatide two times per day ( $5 \mathrm{mcg}$ for 4 weeks, $10 \mathrm{mcg}$ subsequently).

After 28 weeks, patients entered a non-randomized 24-week extension for a total of 1 year of treatment. During this extension phase, patients who were originally randomized to exenatide two times per day were switched to exenatide QWS-AI (2mg), while patients originally randomized to exenatide QWS-AI continued at the same dose. Background glucose-lowering therapies remained unchanged. Rescue medication was initiated by the investigator for patients with a fasting plasma glucose (FPG) level of $>15 \mathrm{mmol} / \mathrm{L}$ (from weeks $4-16$ ) or $>13.3 \mathrm{mmol} / \mathrm{L}$ (from weeks 16-28) on two consecutive study visits, or an A1C of $\geq 9 \%$ after week 28 . All records following the initiation of rescue medications were excluded from the analysis.

\section{End points}

Initial study visits occurred at weeks -2 (screening), -1 , and 0 (randomization). Subsequent visits were every 2 weeks from weeks 2 to 12 , every 4 weeks from weeks 16 to 36 , and every 8 weeks from weeks 44 to 52 . Efficacy and safety measures during the extension phase (weeks 28-52) were identical to those previously described. ${ }^{8}$ Certain safety end points, including cardiovascular events, pancreatitis, and malignancies (ie, thyroid neoplasms and pancreatic cancer) were adjudicated by committee using prespecified criteria.

\section{Statistical analysis}

Efficacy analyses were reported in the modified intentionto-treat (ITT) population, which included all randomized patients who received at least one dose of study drug. Safety was assessed in the treated population, defined as all patients who received at least one dose of study medication, including those who had not been randomized. 
Efficacy measures were summarized descriptively by treatment groups, with means and SEs calculated for all study visits, in addition to changes from baseline to weeks 28 and 52 , with the exception of the analysis of the proportion of patients achieving A1C goals ( $7.0 \%$ and $6.5 \%$, respectively), for which frequency tables were generated. For analysis of efficacy measures by treatment group, the last observation carried forward method was used for missing data. Mixedeffect model with repeated measures (MMRM) was used to estimate the difference and the $95 \%$ CI between treatment groups. No formal hypothesis testing was conducted; therefore, all $\mathrm{p}$ values reported were considered nominal.

For adverse events (AEs), the incidences of overall, serious, and most common AEs $(\geq 5 \%)$ during the initial 28-week randomized period and the 24-week extension were calculated for each treatment group. The incidences of hypoglycemic events were further described by sulfonylurea use or non-use at screening.

\section{RESULTS}

\section{Patient characteristics}

Of the 377 patients randomized, 315 patients completed 28 weeks of treatment with exenatide QWS-AI $(n=197)$ or exenatide two times per day $(n=118)$, all of whom were eligible for the extension phase (figure 1). The 24-week extension was completed by 173 patients who received exenatide QWS-AI for the entire study and 102 patients who switched from exenatide two times per day to exenatide QWS-AI. During the extension phase, 40 patients withdrew from the study, $24(12.2 \%)$ in the exenatide QWS-AI group and 16
$(13.6 \%)$ in the group switching from exenatide two times per day to exenatide QWS. The most common reason for discontinuation in both groups was withdrawal of consent by the patient, which was the most frequent because of the occurrence of an $\mathrm{AE}$ or relocation to another area or state (online supplementary table S1). Treatment compliance was high for both groups (see online supplementary data for more details).

Patient demographic and baseline characteristics for the modified ITT population have been previously published. ${ }^{8}$ At baseline, the treatment groups were generally well balanced. Of the 375 patients in the modified ITT population, $74 \%$ were white and $64 \%$ were male, and the mean age was 56 years. The mean duration of diabetes was 8.5 years, and the mean baseline $\mathrm{A} 1 \mathrm{C}$ was $8.5 \%$. At screening, 89 patients (38.9\%) in the group randomized to exenatide QWS-AI and $60(41.1 \%)$ randomized to exenatide two times per day were receiving sulfonylurea, mostly in combination with metformin.

Rescue medications were initiated in six patients $(4.1 \%)$ originally randomized to exenatide two times per day and five patients $(2.2 \%)$ originally randomized to exenatide QWS-AI during the initial 28-week treatment period. Over the 52-week assessment period, rescue medications were initiated in 22 patients (18.6\%) in the group switching from exenatide two times per day to exenatide QWS-AI and 20 patients (10.2\%) in the group who continued treatment with exenatide QWS-AI. Background antidiabetic therapeutic regimens were similar between the exenatide two times per day and exenatide QWS treatment groups

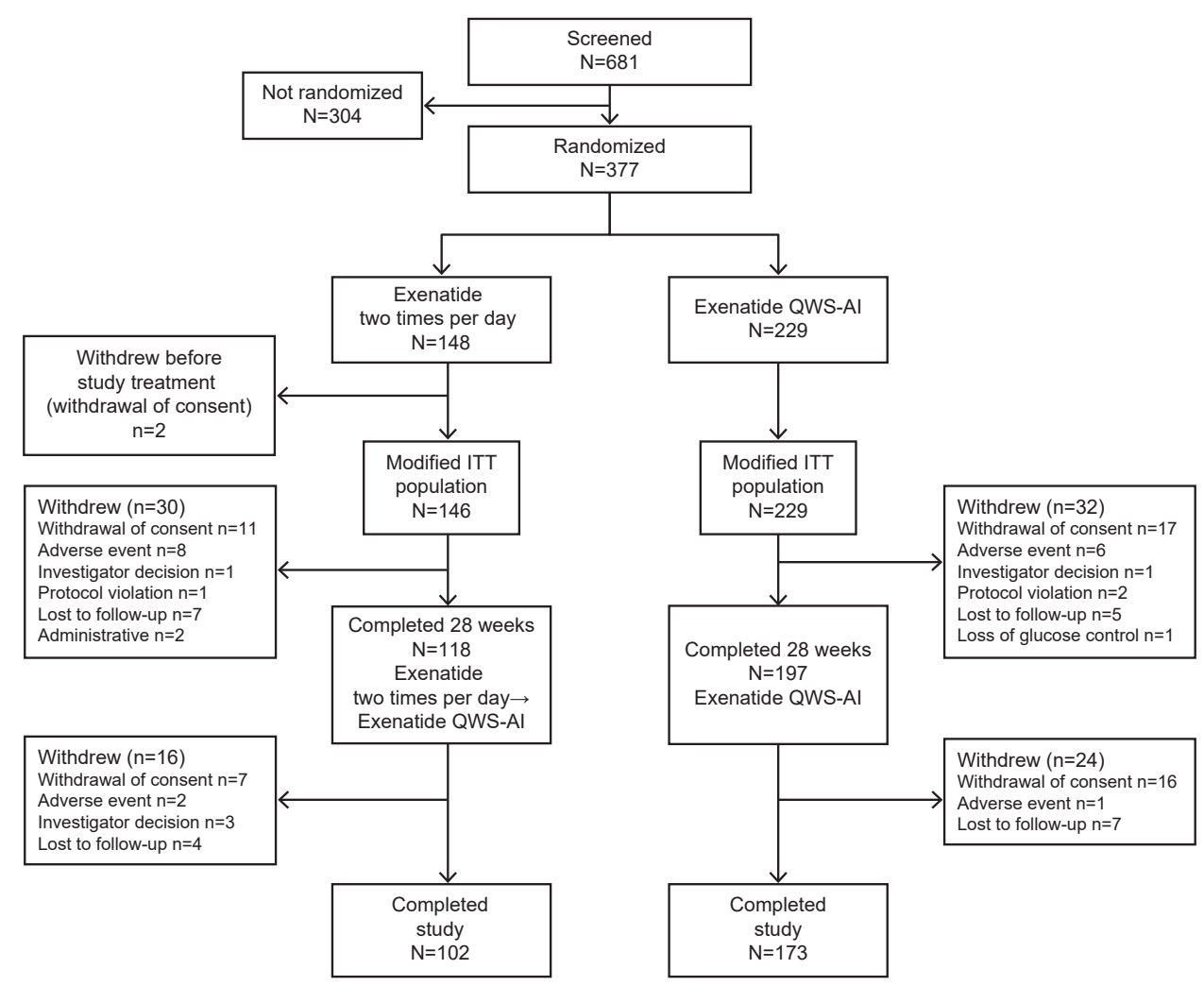

Figure 1 Patient disposition. ITT, intention to treat; QWS-AI, once-weekly suspension by autoinjector. 
during the assessment period of 28 and 52 weeks: biguanides (exenatide two times per day vs exenatide QWS for 28 weeks; 121 patients $(83 \%)$ vs 182 patients $(80 \%)$ and exenatide two times per day vs exenatide QWS for 52 weeks; 124 patients $(84.9 \%)$ vs 185 patients $(80.8 \%))$; sulfonamides (60 patients $(41 \%)$ vs 83 patients $(36 \%)$ and 66 patients $(45.2 \%)$ vs 87 patients $(38.0 \%)$, respectively); and thiazolidinediones (6 patients $(4 \%)$ vs 11 patients $(5 \%)$ and 7 patients (4.8\%) vs 13 patients (5.7\%), respectively).

\section{Efficacy}

Efficacy in patients switching from exenatide two times per day to exenatide QWS-Al

During the initial randomized treatment period, the mean \pm SE reduction in $\mathrm{A} 1 \mathrm{C}$ after 28 weeks of treatment with exenatide two times per day was $-1.1 \% \pm 0.1 \%$, which was less than the reduction of $-1.4 \% \pm 0.1 \%$ observed in the exenatide QWS-AI group (figure 2A). An MMRM analysis of change in $\mathrm{A} 1 \mathrm{C}$ from baseline showed a mean difference of -0.41 (95\% CI -0.68 to -0.14 ) at week 28 (nominal $\mathrm{p}=0.0029$ ) for QWS-AI versus exenatide two times per day. During the extension phase, patients switching from exenatide two times per day to exenatide QWS-AI had further overall reductions in $\mathrm{A} 1 \mathrm{C}$ of approximately $-0.5 \%$ to a mean $\mathrm{A} 1 \mathrm{C}$ of $6.8 \%$ at week 52 . While there was a numerical increase in A1C between weeks 28 and 32, the overlap in $95 \% \mathrm{CI}$ (by MMRM method) between weeks 28 and 32 suggested that there was no meaningful change in A1C from week 28 to 32 in both groups; adjusted mean change in A1C from baseline: -1.45 (95\% CI -1.65 to -1.25$)$ at week 28 vs -1.35 (95\% CI -1.56 to -1.14$)$ at week 32 for
QWS-AI and $-1.04(95 \% \mathrm{CI}-1.28$ to -0.80$)$ at week 28 vs $-0.79(95 \%$ CI -1.03 to -0.55$)$ at week 32 for exenatide two times per day. After week 32, A1C gradually decreased through week 44. After 52 weeks of treatment, the leastsquares mean (LSM) (SE) change in A1C from baseline among all randomized patients who switched from exenatide two times per day was $-1.4 \% \pm 0.1 \%$, which was comparable with that among patients who received exenatide QWS-AI for 52 weeks $(-1.3 \% \pm 0.1 \%)$. An MMRM analysis of change in A1C from baseline showed a mean difference of -0.02 (95\% CI -0.33 to 0.29$)$ at week 52 (nominal $\mathrm{p}=0.9069$ ) for exenatide QWS versus exenatide two times per day.

The proportions of patients who achieved an A1C of $<7.0 \%$ or an $\mathrm{A} 1 \mathrm{C}$ of $\leq 6.5 \%$ at week 52 after switching from exenatide two times per day to exenatide QWS-AI were similar to those among patients who continued exenatide QWS-AI treatment for 52 weeks (figure 2B).

In a similar pattern to A1C, between weeks 28 and 32, while there was a numerical increase in FPG, the overlap in 95\% CI (by the MMRM method) suggested that there was no meaningful change in FPG between weeks 28 and 32 in either group; adjusted mean change from baseline: -31.42 (95\% CI -39.10 to -23.73$)$ at week 28 vs -21.01 (95\% CI -28.79 to -13.24$)$ at week 32 for QWS-AI and $-20.02(95 \%$ CI -29.73 to -10.31$)$ at week 28 vs $-7.48(95 \%$ CI -17.31 to 2.35) at week 32 for exenatide two times per day. After week 32, reductions in FPG were observed, from a mean of $8.6 \pm 0.3 \mathrm{mmol} / \mathrm{L}$ at week 28 to $7.5 \pm 0.2 \mathrm{mmol} / \mathrm{L}$ at week 52 (figure 2C).
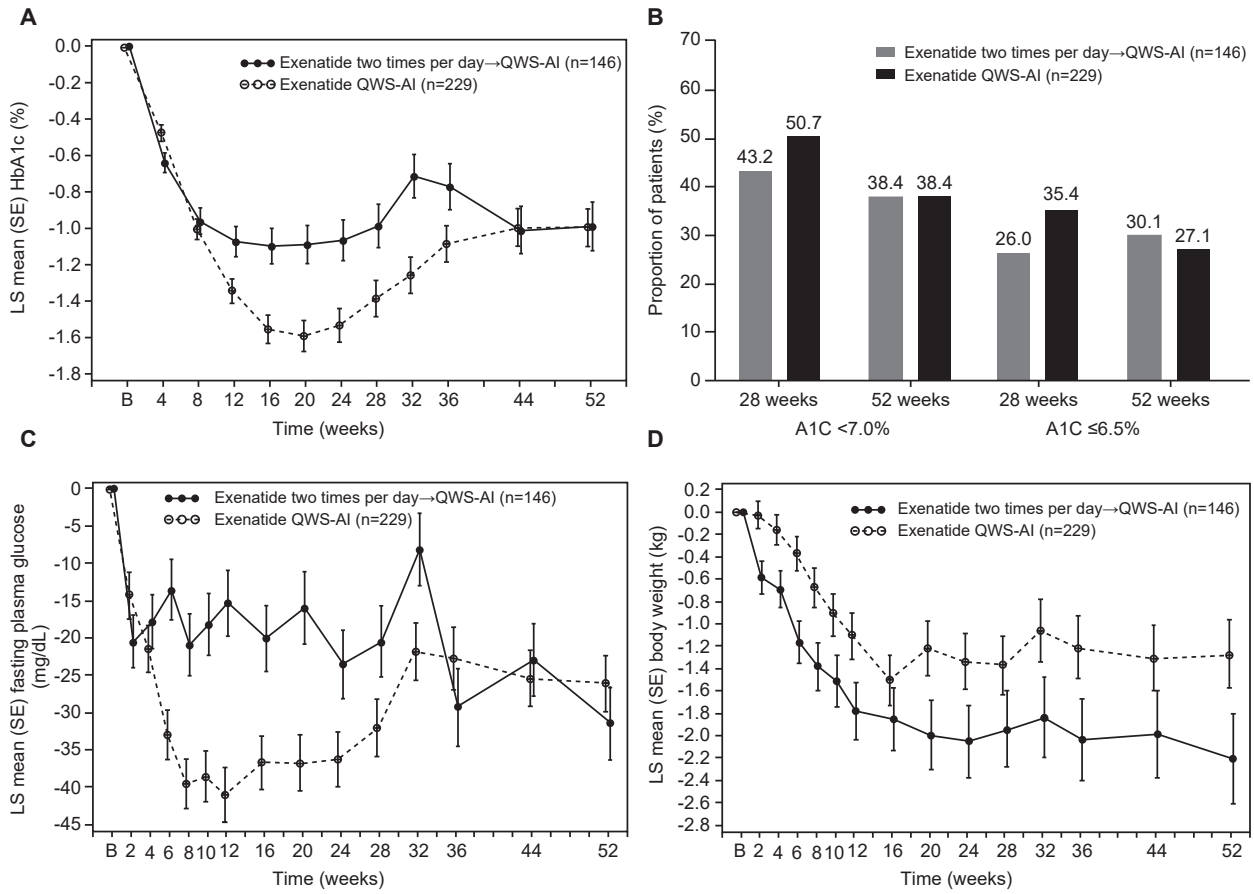

Figure 2 Effects of switching from exenatide two times per day to exenatide QWS-Al or continuing exenatide QWS-AI on various measures of efficacy in the modified intention-to-treat population. (A) A1C over time. (B) Proportion of patients achieving A1C goals at weeks 28 and 52. (C) Fasting plasma glucose over time. (D) Body weight over time. A1C, glycated hemoglobin; LS, least squares; QWS-AI, once-weekly suspension by autoinjector. 
Body-weight reductions were achieved during 28 weeks of treatment with exenatide two times per day, and although the mean body weight increased at week 44 after switching to exenatide QWS-AI, clinically relevant reductions were still observed at week 52 (figure 2D). The mean reduction in body weight from baseline was greater for patients who switched from exenatide two times per day to exenatide QWS-AI than for those who received exenatide QWS-AI for 52 weeks $(-2.6 \pm 0.4 \mathrm{~kg}$ and $-1.5 \pm 0.3 \mathrm{~kg}$, respectively). An MMRM analysis of change in body weight from baseline showed a mean difference of 0.55 (95\% CI -0.27 to 1.36 ) at week 28 (nominal $\mathrm{p}=0.1862)$ and 0.92 (95\% CI -0.04 to 1.89) at week 52 (nominal $\mathrm{p}=0.0599$ ) for exenatide QWS versus exenatide two times per day.

After 52 weeks, there was a small change in systolic blood pressure (SBP) among patients who switched from exenatide two times per day to exenatide QWS-AI (online supplementary table S3). Decreases in SBP from baseline were initially observed between weeks 0 and 28 (mean change of $-3.0 \mathrm{~mm}$ $\mathrm{Hg}$ ), but thereafter, SBP increased (mean change of $-1.3 \mathrm{~mm}$ $\mathrm{Hg}$ at week 52). There were also no clinically meaningful changes in fasting lipids or other cardiovascular risk factors, including high-sensitivity C-reactive protein, brain natriuretic peptide, or urinary albumin:creatinine ratio (online supplementary table S3). A small increase in heart rate from baseline was observed in the exenatide two times per day group during the 28-week controlled period (mean change of +1.6 beats $/ \mathrm{min}$ ). During the extension phase, a further increase in heart rate was observed after patients switched to exenatide QWS-AI (mean change from baseline to week 52 of +4.3 beats $/ \mathrm{min}$; online supplementary table $\mathrm{S} 3$ ).

\section{Efficacy in patients continuing treatment with exenatide QWS-Al}

Among patients who continued treatment with exenatide QWS-AI for 52 weeks, reductions in A1C and FPG achieved during the randomized treatment period were maintained during the extension phase. After 28 weeks, the mean reduction in $\mathrm{A} 1 \mathrm{C}$ among patients randomized to exenatide QWS-AI was greater than that observed among patients randomized to exenatide two times per day (as described previously; figure 2A). Between weeks 28 and 52, A1C stabilized in the group continuing treatment with exenatide QWS-AI (figure 2A). After 52 weeks of treatment, the mean change in A1C from baseline among patients continuing exenatide QWS-AI treatment was $-1.3 \% \pm 0.1 \%$ (mean A1C of $7.0 \%$ at week 52 ).

FPG levels remained stable in patients who received exenatide QWS-AI from randomization, in contrast to the overall reductions in FPG after switching from exenatide two times per day to exenatide QWS-AI (described previously), with little difference in levels at week 28 $(7.9 \pm 0.2 \mathrm{mmol} / \mathrm{L})$ and week $52 \quad(7.9 \pm 0.2 \mathrm{mmol} / \mathrm{L})$ (figure 2C).

Reductions in body weight during randomized treatment with exenatide QWS-AI were sustained with continued treatment through week 52 (figure 2D). After 52 weeks, body weight was reduced by a mean of $-1.5 \pm 0.3 \mathrm{~kg}$.
There was a mean change of $-1.1 \mathrm{~mm} \mathrm{Hg}$ in SBP from baseline to week 52 among patients who continued exenatide QWS-AI treatment (online supplementary table S3). Fasting lipids or other cardiovascular risk factors showed no clinically meaningful changes with continued exenatide QWS-AI treatment (online supplementary table S3). Heart rate was slightly increased from baseline at week 28 (mean change of +1.9 beats $/ \mathrm{min}$ ), but no further increases were observed between weeks 28 and 52 (mean change from baseline to week 52 of +1.5 beats $/ \mathrm{min}$; online supplementary table S3).

\section{Safety}

Treatment with exenatide QWS-AI was well tolerated during the extension phase, with no unexpected changes in the safety and tolerability profile among patients switching from exenatide two times per day to exenatide QWS-AI or among those continuing treatment with exenatide QWSAI. The proportion of patients with AEs was lower during the extension phase than during the first 28 weeks in both groups (table 1). No AEs occurred in $\geq 5 \%$ of patients in either group during the extension phase.

The most common AEs during the 28-week controlled period were nausea and diarrhea, with incidences of $20.5 \%$ and $11.6 \%$, respectively, with exenatide two times per day and $9.6 \%$ and $5.2 \%$, respectively, with exenatide QWS-AI (table 1). Among patients switching from exenatide two times per day to exenatide QWS-AI, the incidences of both nausea $(4.3 \%)$ and diarrhea $(1.7 \%)$ were lower during the extension phase (weeks 28-52) than during randomized treatment with exenatide two times per day, but remained higher than that observed in patients who continued with exenatide QWS-AI (table 1).

Injection site nodules occurred in a smaller proportion of patients during the extension phase $(1.9 \%)$ than during the 28-week controlled period $(8.0 \%)$ in the overall safety population (table 1). The proportion of patients who reported injection site nodules in the exenatide two times per day group increased from $0.7 \%$ during randomized treatment to $4.3 \%$ after switching to exenatide QWS-AI in the 24-week extension period (table 1). In contrast, among the group continuing exenatide QWS-AI treatment, the proportion of patients with injection site nodules decreased from $12.7 \%$ during the first 28 weeks of treatment to $0.5 \%$ during the extension phase (table 1).

No major hypoglycemic events occurred during the 28-week controlled period or the 24-week extension in either treatment group (table 1). Minor hypoglycemic events occurred more frequently during weeks 0-28 than during weeks 28-52 in both treatment groups and was more often associated with concomitant sulfonylurea use (table 1). The incidence of minor hypoglycemic events was lower with exenatide two times per day than exenatide QWS-AI during the 28-week controlled period among patients with concomitant sulfonylurea use, but was $8.0 \%$ and $8.1 \%$ in the respective groups during the 24-week extension period. 
Table 1 AEs occurring during the controlled treatment period (weeks 0-28) and extension phase (weeks 28-52)

\begin{tabular}{|c|c|c|c|c|}
\hline & \multicolumn{2}{|c|}{ Exenatide two times per day $\rightarrow$ QWS-AI } & \multicolumn{2}{|c|}{ Exenatide QWS-AI } \\
\hline & $\begin{array}{l}0-28 \text { weeks } \\
(n=146)\end{array}$ & $\begin{array}{l}28-52 \text { weeks } \\
(n=116)\end{array}$ & $\begin{array}{l}0-28 \text { weeks } \\
(n=229)\end{array}$ & $\begin{array}{l}\text { 28-52 weeks } \\
(n=193)\end{array}$ \\
\hline All patients with AEs & $108(74.0)$ & $64(55.2)$ & $162(70.7)$ & 95 (49.2) \\
\hline Patients with serious AEs & $7(4.8)$ & $6(5.2)$ & $6(2.6)$ & $7(3.6)$ \\
\hline $\begin{array}{l}\text { Patients with AEs leading to } \\
\text { withdrawal }\end{array}$ & $11(7.5)$ & $2(1.7)$ & $11(4.8)$ & $2(1.0)$ \\
\hline $\begin{array}{l}\text { Patients with AEs leading to } \\
\text { death }\end{array}$ & 0 & $1(0.9)$ & 0 & 0 \\
\hline \multicolumn{5}{|l|}{ AEs occurring in $\geq 5 \%$ of patients } \\
\hline Nausea & $30(20.5)$ & $5(4.3)$ & $22(9.6)$ & $1(0.5)$ \\
\hline Injection site nodule & $1(0.7)$ & $5(4.3)$ & $29(12.7)$ & $1(0.5)$ \\
\hline Diarrhea & $17(11.6)$ & $2(1.7)$ & $12(5.2)$ & $3(1.6)$ \\
\hline Headache & $9(6.2)$ & 0 & $13(5.7)$ & $2(1.0)$ \\
\hline $\begin{array}{l}\text { Upper respiratory tract } \\
\text { infection }\end{array}$ & $5(3.4)$ & $3(2.6)$ & $13(5.7)$ & $2(1.0)$ \\
\hline Vomiting & $9(6.2)$ & $2(1.7)$ & $8(3.5)$ & $1(0.5)$ \\
\hline \multicolumn{5}{|l|}{ Hypoglycemia* } \\
\hline $\begin{array}{l}\text { With concomitant sulfonylurea } \\
\text { use at screening }\end{array}$ & $n=60$ & $n=50$ & $\mathrm{n}=89$ & $\mathrm{n}=74$ \\
\hline Major hypoglycemia & 0 & 0 & 0 & 0 \\
\hline Minor hypoglycemia & $11(18.3)$ & $4(8.0)$ & $22(24.7)$ & $6(8.1)$ \\
\hline Mild & $5(8.3)$ & $4(8.0)$ & $13(14.6)$ & $5(6.8)$ \\
\hline Moderate & $6(10.0)$ & 0 & $9(10.1)$ & $1(1.4)$ \\
\hline Severe & 0 & 0 & 0 & 0 \\
\hline Symptoms of hypoglycemia & $15(25.0)$ & $10(20.0)$ & $24(27.0)$ & $9(12.2)$ \\
\hline Mild & $12(20.0)$ & $9(18.0)$ & $20(22.5)$ & $7(9.5)$ \\
\hline Moderate & $2(3.3)$ & $1(2.0)$ & $4(4.5)$ & $2(2.7)$ \\
\hline Severe & $1(1.7)$ & 0 & 0 & 0 \\
\hline $\begin{array}{l}\text { Without concomitant } \\
\text { sulfonylurea use at screening }\end{array}$ & $n=86$ & $n=66$ & $n=140$ & $\mathrm{n}=119$ \\
\hline Major hypoglycemia & 0 & 0 & 0 & 0 \\
\hline Minor hypoglycemia & $3(3.5)$ & $3(4.5)$ & $3(2.1)$ & $2(1.7)$ \\
\hline Mild & $1(1.2)$ & $2(3.0)$ & $2(1.4)$ & 0 \\
\hline Moderate & $2(2.3)$ & $1(1.5)$ & $1(0.7)$ & $2(1.7)$ \\
\hline Severe & 0 & 0 & 0 & 0 \\
\hline Symptoms of hypoglycemia & $5(5.8)$ & $2(3.0)$ & $12(8.6)$ & $5(4.2)$ \\
\hline Mild & $4(4.7)$ & $2(3.0)$ & $11(7.9)$ & $4(3.4)$ \\
\hline Moderate & $1(1.2)$ & 0 & $1(0.7)$ & $1(0.8)$ \\
\hline Severe & 0 & 0 & 0 & 0 \\
\hline
\end{tabular}

Data are presented as $\mathrm{n}(\%)$.

*The following hypoglycemia definitions were used: major, event resulting in loss of consciousness, seizure, or coma (or other mental status change consistent with neuroglycopenia) that resolved after glucose or glucagon administration, or any event requiring third-party assistance to resolve due to severe impairment of consciousness or behavior associated with glucose concentration $<3 \mathrm{mmol} / \mathrm{L}(54 \mathrm{mg} / \mathrm{dL})$; $\mathrm{minor}$, $\mathrm{non}-$ major hypoglycemia event with symptoms consistent with hypoglycemia and glucose concentration $<3 \mathrm{mmol} / \mathrm{L}(54 \mathrm{mg} / \mathrm{dL})$ prior to treatment; symptoms of hypoglycemia, events not meeting the criteria for major or minor hypoglycemic events.

$\mathrm{AE}$, adverse event; $\mathrm{QWS}-\mathrm{Al}$, once-weekly suspension by autoinjector. 
The incidence of serious AEs was higher among patients in the exenatide two times per day group than in those in the exenatide QWS-AI group, both during the 28-week controlled period and after switching to exenatide QWS-AI in the 24-week extension period (table 1). Two serious AEs of pancreatitis were reported in the exenatide QWS-AI group (one during the 28-week controlled period and one during the extension phase); both were adjudicated as pancreatitis and assessed by the investigator to be study related. One patient in the exenatide two times per day group developed pancreatic cancer, which was reported approximately 6 weeks after the 10-week post-treatment follow-up visit; this event was adjudicated and assessed as related to study treatment by the investigator. Four cardiovascular events were reported during the study and were adjudicated; none were assessed by the investigator as being study related. In the exenatide QWS-AI group, myocardial infarction occurred in one patient during the 28-week controlled period, and acute myocardial infarction and coronary artery disease occurred in one patient each during the extension phase. In the exenatide two times per day group, one patient had atrial fibrillation during the extension period after switching to exenatide QWSAI. Among patients with available antibody data at week $52,61.8 \%$ of patients who switched treatment from exenatide two times per day to exenatide QWS-AI and $51.5 \%$ of patients who received exenatide QWS-AI throughout the study were positive for exenatide antibodies. The majority of these patients had low titers (online supplementary table S4).

\section{DISCUSSION}

Since the approval of exenatide two times per day, the first-in-class GLP-1RA, longer-acting GLP-1RAs have been developed with reduced frequency of administration and, in some cases, simplified injection devices that improve convenience and ease of use. ${ }^{7}$ One such product is exenatide QWS-AI, a long-acting, soluble formulation of exenatide administered using an autoinjector, which was compared with exenatide two times per day over 28 weeks in the DURATION-NEO-1 study. ${ }^{8}$

This 24-week extension of DURATION-NEO-1 demonstrated that patients can be switched from exenatide two times per day to exenatide QWS-AI simply and safely, and that improvements in glycemic control during 28 weeks of treatment with exenatide QWS-AI are maintained through 52 weeks. Patients who switched treatment had further glycemic improvements to a mean A1C of $6.8 \%$ and a mean FPG of $7.5 \mathrm{mmol} / \mathrm{L}$ at week 52 , despite transient increases in A1C and FPG after switching to exenatide QWS-AI, most likely reflecting the time taken to reach steady state with the extended-release formulation (approximately 8 weeks). The proportion of patients achieving A1C targets at week 52 was comparable with that in the group receiving exenatide QWS-AI for the entire study period. Comparable improvements in body weight from baseline to week 52 were also seen in both treatment groups. In both treatment groups, few patients required initiation of rescue medication, although the proportion of patients needing rescue medication was lower in the group continuing exenatide QWS-AI for 52 weeks than in the group switching from exenatide two times per day to exenatide QWS-AI.

While the DURATION-NEO-1 study ended at 52 weeks, the beneficial effects of switching treatment to exenatide QW (aqueous) were demonstrated in long-term extensions of both the DURATION-1 and DURATION-3 clinical studies. ${ }^{9-13}$ In the DURATION-1 study, sustained benefits from exenatide QW (aqueous) were observed for up to 7 years in responders who remained in the study and continued therapy. ${ }^{11} 12$ Those responders who continued treatment with exenatide QW (aqueous) for 7 years maintained clinically significant improvements from baseline in A1C (LSM change of $-1.5 \%)$, FPG $(-1.3 \mathrm{mmol} / \mathrm{L})$, and body weight $(-3.9 \mathrm{~kg}){ }^{12}$ Sustained beneficial effects were also observed after 3 years in the DURATION-3 study, with improvements from baseline in A1C, fasting serum glucose, and body weight (LSM changes of $-1.0 \%,-1.7 \mathrm{mmol} / \mathrm{L}$, and $-2.5 \mathrm{~kg}$, respectively); $40 \%$ of patients maintained an $\mathrm{A} 1 \mathrm{C}$ of $<7.0 \%{ }^{10}$

In the current study, switching from exenatide two times per day to exenatide QWS-AI was well tolerated, with no unexpected changes in the AE profile. As expected, the incidence of injection site nodules increased after switching from exenatide two times per day to exenatide QWS-AI. Injection site nodules, which are usually transient and resolve without intervention, are consistent with the properties of the microspheres used in the QW formulation of exenatide $^{6}$; higher incidences of nodules were observed in studies that compared microsphere-encapsulated exenatide QW and exenatide two times per day. ${ }^{14}{ }^{15}$ Interestingly, the proportion of patients in the current study who reported injection site nodules after switching from exenatide two times per day to exenatide QWS-AI in the extension phase was lower than that of patients who received exenatide QWS-AI during the initial 28-week treatment period. Consistent with other studies of exenatide QW (aqueous), ${ }^{11}{ }^{16}$ small increases in heart rate from baseline to week 52 were observed in the current study.

This study has some limitations. The open-label design may have contributed to bias in the study conduct and patient behaviors. Due to the high discontinuation rate, the populations of the two treatment groups in the extension phase could no longer be considered balanced as they had been during the 28-week controlled treatment period. Therefore, we were unable to analyze the 52-week data using the MMRM analysis used for the week 28 data, ${ }^{8}$ so data were analyzed descriptively.

\section{CONCLUSIONS}

Switching from exenatide two times per day to exenatide QWS-AI after 28 weeks of treatment was associated with further improvements in glycemic control and reduced body weight without additional safety concerns over a 24-week extension period in patients with type 2 diabetes. 
Those who continued treatment with exenatide QWS-AI for 52 weeks maintained the improvements in glycemic measures and body weight seen at week 28. Consistent with the properties of the microspheres used in the QW formulation, the incidence of injection site nodules, the majority of which were mild in intensity, increased after switching from exenatide two times per day to exenatide QWS-AI. These results support long-term use of exenatide QWS-AI and switching from exenatide two times per day to exenatide QWS-AI for patients who respond to exenatide two times per day therapy but are seeking an easier delivery system and treatment with comparable efficacy and tolerability, with the potential for improved long-term treatment adherence and persistence.

\author{
Author affiliations \\ ${ }^{1}$ Section of Endocrinology and Metabolism, MultiCare Rockwood Clinic, Spokane, \\ Washington, USA \\ ${ }^{2}$ Dallas Diabetes Research Center at Medical City, Dallas, Texas, USA \\ ${ }^{3}$ Bristol-Myers Squibb, Princeton, New Jersey, USA \\ ${ }^{4}$ AstraZeneca, Gaithersburg, Maryland, USA \\ ${ }^{5}$ Late clinical development, AstraZeneca, Gaithersburg, Maryland, USA \\ ${ }^{6}$ Clinical, Diabetes, Metabolism and Gl, AstraZeneca, Gaithersburg, Maryland, USA
}

Acknowledgements The authors thank the patients for their participation in this study, as well as the study investigators and contributors from each site. The authors also thank Peter Öhman, MD, PhD, of AstraZeneca, Gaithersburg, Maryland, USA, for his contributions to the study design and conduct, data analysis and interpretation, and development of the initial draft of this article. Sarah Greig and Raewyn Poole of inScience Communications, Springer Healthcare, provided medical writing support in accordance with Good Publication Practice and funded by AstraZeneca.

Contributors CHW, JR, MLV, and NI contributed to the outline of the manuscript and were involved in the writing, discussion, and critical revision of the manuscript, study conduct, and analysis and interpretation of the data. HW contributed to the statistical analysis of the manuscript and the writing, discussion, and critical revision of the manuscript. EH contributed to the outline of the manuscript and was involved in the writing, discussion, and critical revision of the manuscript and the analysis and interpretation of the data.

Funding The DURATION-NE0-1 study was supported by Bristol-Myers Squibb and AstraZeneca. Development of this manuscript was supported by AstraZeneca.

Competing interests CHW is an advisor, speaker, and consultant for AstraZeneca, Boehringer Ingelheim, Eli Lilly, Janssen, Novo Nordisk, and Sanofi, an advisor for Abbott, and a speaker for Insulet; JR has served on scientific advisory boards and received honoraria or consulting fees from Eli Lilly, Novo Nordisk, Sanofi, Janssen, Boehringer Ingelheim, and Intarcia Therapeutics, and has received grants/research support from Merck, Pfizer, Sanofi, Novo Nordisk, Bristol-Myers Squibb, Eli Lilly, GlaxoSmithKline, AstraZeneca, Janssen, Genentech, Boehringer Ingelheim, Intarcia Therapeutics, and Lexicon; MLV was a full-time employee of Bristol-Myers Squibb during the time the study was conducted and completed; $\mathrm{EH}$ is an employee of AstraZeneca; HW was a contractor for AstraZeneca during the time the statistical analyses were done; $\mathrm{NI}$ is an employee of AstraZeneca.

Patient consent for publication Not required.

Ethics approval The study protocol was approved at each study site by the appropriate institutional review board (main study site IRB approval ID MED1-12198 and MED1-12-389), and the study was conducted in accordance with the Declaration of Helsinki. All patients provided written informed consent prior to participation.

Provenance and peer review Not commissioned; externally peer reviewed.
Data availability statement Data can be made available upon request. Data underlying the findings described in this manuscript may be obtained in accordance with AstraZeneca's data sharing policy described online (https://astr azenecagrouptrials. pharmacm.com/ST/Submission/Disclosure).

Open access This is an open access article distributed in accordance with the Creative Commons Attribution Non Commercial (CC BY-NC 4.0) license, which permits others to distribute, remix, adapt, build upon this work non-commercially, and license their derivative works on different terms, provided the original work is properly cited, appropriate credit is given, any changes made indicated, and the use is non-commercial. See: http://creativecommons.org/licenses/by-nc/4.0/.

ORCID iD

Carol H Wysham http://orcid.org/0000-0002-3056-0047

\section{REFERENCES}

1 Polonsky WH, Henry RR. Poor medication adherence in type 2 diabetes: recognizing the scope of the problem and its key contributors. Patient Prefer Adherence 2016;10:1299-307.

2 Alatorre C, Fernández Landó L, Yu M, et al. Treatment patterns in patients with type 2 diabetes mellitus treated with glucagon-like peptide-1 receptor agonists: higher adherence and persistence with dulaglutide compared with once-weekly exenatide and liraglutide. Diabetes Obes Metab 2017;19:953-61.

3 Asche C, LaFleur J, Conner C. A review of diabetes treatment adherence and the association with clinical and economic outcomes. Clin Ther 2011;33:74-109.

4 Jha AK, Aubert RE, Yao J, et al. Greater adherence to diabetes drugs is linked to less hospital use and could save nearly $\$ 5$ billion annually. Health Aff (Millwood) 2012;31:1836-46.

5 Stuart BC, Simoni-Wastila L, Zhao L, et al. Increased persistency in medication use by U.S. Medicare beneficiaries with diabetes is associated with lower hospitalization rates and cost savings. Diabetes Care 2009;32:647-9.

6 DeYoung MB, MacConell L, Sarin V, et al. Encapsulation of exenatide in poly-(D,L-lactide-co-glycolide) microspheres produced an investigational long-acting once-weekly formulation for type 2 diabetes. Diabetes Technol Ther 2011;13:1145-54

7 LaRue S, Springer J, Noderer M, et al. Evaluation of the use of exenatide once-weekly suspension autoinjector among patients with type 2 diabetes mellitus and health care professionals. J Diabetes Sci Technol 2019;13:226-34.

8 Wysham $\mathrm{CH}$, Rosenstock J, Vetter ML, et al. Efficacy and tolerability of the new autoinjected suspension of exenatide once weekly versus exenatide twice daily in patients with type 2 diabetes. Diabetes Obes Metab 2018;20:165-72.

9 Buse JB, Drucker DJ, Taylor KL, et al. DURATION-1: exenatide once weekly produces sustained glycemic control and weight loss over 52 weeks. Diabetes Care 2010;33:1255-61.

10 Diamant M, Van Gaal L, Guerci B, et al. Exenatide once weekly versus insulin glargine for type 2 diabetes (DURATION-3): 3year results of an open-label randomised trial. Lancet Diabetes Endocrinol 2014;2:464-73.

11 Henry RR, Klein EJ, Han J, et al. Efficacy and tolerability of exenatide once weekly over 6 years in patients with type 2 diabetes: an uncontrolled open-label extension of the DURATION-1 study. Diabetes Technol Ther 2016;18:677-86.

12 Philis-Tsimikas A, Wysham CH, Hardy E, et al. Efficacy and tolerability of exenatide once weekly over 7 years in patients with type 2 diabetes: an open-label extension of the DURATION-1 study. $J$ Diabetes Complications 2019;33:223-30.

13 Wysham C, Bergenstal R, Malloy J, et al. DURATION-2: efficacy and safety of switching from maximum daily sitagliptin or pioglitazone to once-weekly exenatide. Diabet Med 2011;28:705-14.

14 Drucker DJ, Buse JB, Taylor K, et al. Exenatide once weekly versus twice daily for the treatment of type 2 diabetes: a randomised, openlabel, non-inferiority study. Lancet 2008;372:1240-50.

15 Blevins T, Pullman J, Malloy J, et al. DURATION-5: exenatide once weekly resulted in greater improvements in glycemic control compared with exenatide twice daily in patients with type 2 diabetes. J Clin Endocrinol Metab 2011;96:1301-10.

16 Holman RR, Bethel MA, Mentz RJ, et al. Effects of once-weekly exenatide on cardiovascular outcomes in type 2 diabetes. $N$ Engl $J$ Med 2017;377:1228-39. 